\title{
THE IR COLOR EVOLUTION OF CIRCUMSTELLAR SHELLS
}

\author{
B. M. LEWIS \\ N.A.I.C. \\ P.O. Box 995 \\ Arecibo, PR00612 \\ YERVANT TERZIAN \\ N.A.I.C. \\ Cornell University, \\ Ithaca, NY14853.
}

\begin{abstract}
The far IR colors of circumstellar shells are delimited by those with masers; few other objects share the same colors. The resulting (25-12) $\mu \mathrm{m}$ color range is primarily due to secular increases in the mass-loss rate, up to a progenitor-mass dependent maximum. Only the most massive progenitors can sustain the mass-loss rate required by the reddest shells.
\end{abstract}

\section{IR Two-Color Diagram}

Red giants lose mass in a low velocity wind that dust forms in. Dust intercepts all or part of the stellar luminosity, and reradiates it in the far infra-red, where the Infra Red Astronomical Satellite (IRAS) was able to detect it. The heavier the mass loss rate, the thicker the dust shell, and the redder the emergent radiation. Dust also shields embedded molecules against photodissociation by interstellar UV for a time, until prevented by radial dilution. This enables molecules, such as water and $\mathrm{OH}$, to survive into regions where they can mase.

A major consequence of the IRAS Survey, and its ability to detect dusty circumstellar shells, is the delineation of their far-IR signatures. The full color range of oxygen-rich shells is set by success in detecting their masers (Lewis 1990): this distribution is shown in Fig. 1. The plotted IR color range is almost limited to shells with ongoing mass-loss, as few other types of object achieve them (Hacking et al 1985; van der Veen and Habing 1988; Walker et al 1989): only the brightest stellar photospheres were detected by IRAS, and these have colors close to the Rayleigh-Jeans point at position $\mathbf{A}$. This is separated by a distinct (25-12) $\mu \mathrm{m}$ gap from the Mira sequence, in the IR color most closely related to the stellar mass loss rate. The largest group of confusing interlopers are T Tauri stars, which Harris et al (1988) find at (60-25) $\mu \mathrm{m}>-0.68$ and (25-12) $\mu \mathrm{m}$ $>-0.35$. Less than $1 \%$ of alternate identifications are otherwise expected.

We have a well characterized all-sky survey for circumstellar shells from IRAS. This is neither biased to strong sources near the galactic plane, nor to optically thin shells elsewhere. But followup studies on color selected IRAS sources also distinguish a group of objects without well defined periods, and with at most small amplitudes of fluctuation along the righthand margin of the shell distribution. These sometimes have masers too. We consider them to be PPN or proto 
planetary nebulae, as they have expanding "fossil" shells left over from a recently finished massloss phase. These shells are redder than normal as their dust is all at a larger, colder distance. Every star on terminating its mass-loss phase in this sense becomes a PPN, and so traverses the red margin of Fig. 1. The literature on PPN is summarized by Kwok et al (1990).

There is a remaining identification question about the nature of masing nondetections among color selected sources, as these also seem to be circumstellar shells. The Arecibo $1612 \mathrm{MHz}$ survey only identified masers in $\approx 60 \%$ of objects with $-0.6<(25-12) \mu \mathrm{m}<0$ (Lewis et al 1990), a rate that is almost unchanged by a second look. Although a few may be $T$ Tauri stars, there is certainly a residual population of $\mathrm{O}$-rich objects with $(25-12) \mu \mathrm{m}<-0.35$ and no masers. These can in principle be understood as binary systems with widely-separated, degenerate companions. The UV flux from the accretion disk these garner from the red giant wind, and from the white dwarf photosphere, can photodissociate many of their circumstellar molecules from within the shell. This explains the absence of masers from sources where the characteristic IR signature of dust shows there is plenty of shielding for molecules against interstellar UV. Our scenario is supported by a search of the IUE archives, which shows that 12 of the 15 sources there, with no water or $\mathrm{OH}$ emission and IR colors appropriate to a dust-shell, have UV continua. This also explains an earlier finding that Miras with companion stars never have strong masers (Bowers and Hagen 1984). We suspect that most masing nondetections are D(usty)-type symbiotic stars.

To summarize, molecules need the protection of dust for their continued existence in a circumstellar environment. When a star is solitary, it only requires sufficient mass-loss to provide a suitably dusty shell for molecules to exist. As the mass-loss rate increases, the shell's color reddens, and dust shielding is extended to a more distant, colder zone. This is most directly shown by the progressive addition of masers with increasing (25-12) $\mu \mathrm{m}$ color, as the longevity of molecules is increased at larger radii by the increased dust shielding (Lcwis 1990). But when a white dwarf or neutron star companion provides a local, tethered source of UV, dust shielding may be ineffective, and the shell may be stripped of its normal molecular content.

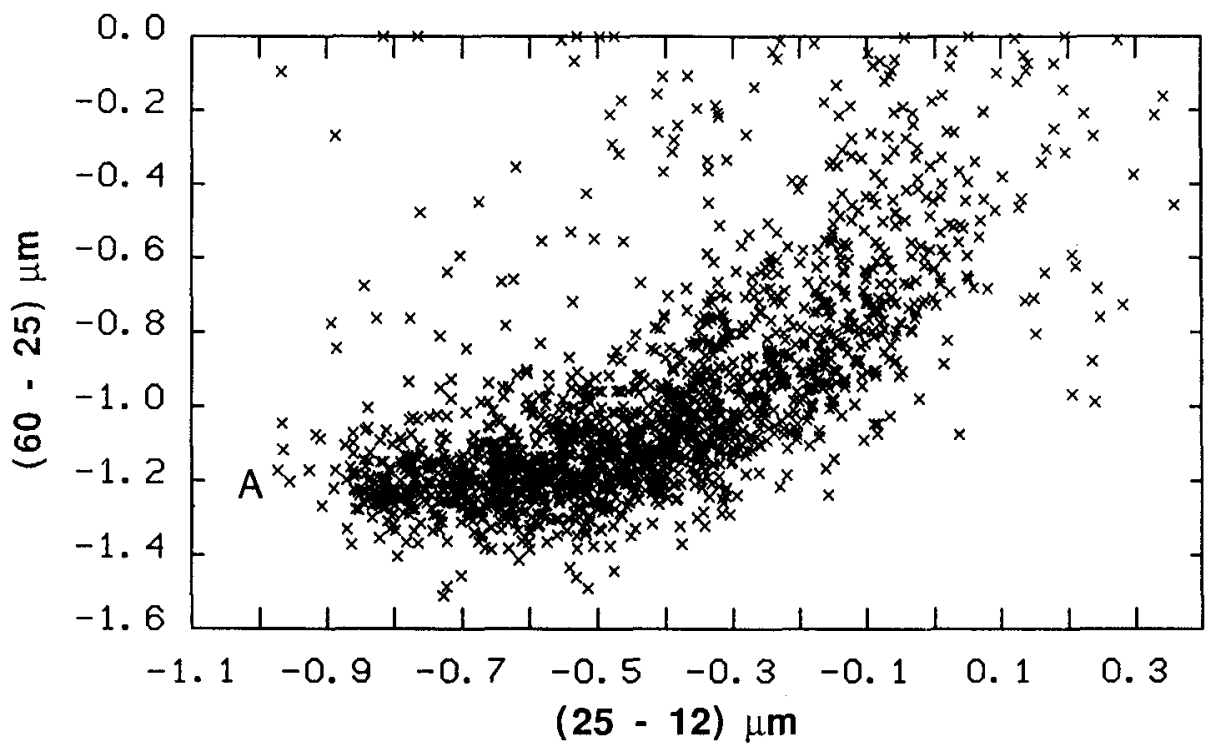

Figure 1: IR two-color diagram of circumstellar shells with detected $\mathrm{SiO}$, water, mainline or $1612 \mathrm{MHz}$ masers, from papers listed in Lewis (1990). The IR colors of photospheres lie to the left of the RayleighJeans point "A": most sources beyond the righthand cdge of the distribution are the "fossil" shclls of PPN. 


\section{Evolving Mass Loss Rates}

The form of Fig. 1 suggests color evolution. While the IR colors of shells with ongoing mass-loss are redder than any photosphere (near position A), they are bluer than the PPN lying along the righthand margin of the shell distribution. Since most stars must evolve through these phases to become white dwarfs, so must their IR colors. The idea that a shell is a result of a gradual increase in mass-loss predates IRAS. It was invoked first as an explanation for the OH / IR star luminosity function (Baud and Habing 1983), and is echoed in van der Veen's (1989) extreme assumption that every star follows the same IR track. This is at variance with the (25-12) $\mu \mathrm{m}$ versus b distribution of OH / IR stars, and with the colors of low progenitor-mass PPN like 18095+2704 (Hrivnak et al 1988). But Bedijn's (1988) simulations show that a canonically rising mass-loss rate is consistent with the distributions of Mira periods and white dwarf masses.

The carbon star two-color diagram provides an independent argument for evolution. Optically recognized C-stars lie in a (60-25) $\mu \mathrm{m}$ sequence above the Rayleigh-Jeans point at $\mathrm{A}$. They have little $\mathrm{CO}$ emission. Mass-losing C-stars on the other hand mimic the M stars. Willems and de Jong (1988) suggest that when an $\mathrm{M}$ star becomes a C-star during a thermal pulse, it is slow to restart its mass-loss, so its colors evolve like those of a "fossil" shell. The (60-25) $\mu \mathrm{m}$ color sequence is then the IR echo of very old, distant, cold dust. The evolutionary path of a shell is shown by the "snapshot" color positions of a sample, just like the HR diagram displays the evolution of a globular cluster population. However Epchtein et al (1990) still prefer to model the carbon-star sequence as a progenitor mass sequence.

A progenitor mass interpretation of the Mira sequence is also suggested by an uncritical acceptance of the color segregation between Miras and OH / IR stars. Miras were optically recognized at large I b I, so most have modest masses and thin, blue shells; OH / IR stars were originally found in scanning radio surveys at small $\mathrm{I} b \mathrm{l}$, which mainly detected strong $\mathrm{OH}$ masers

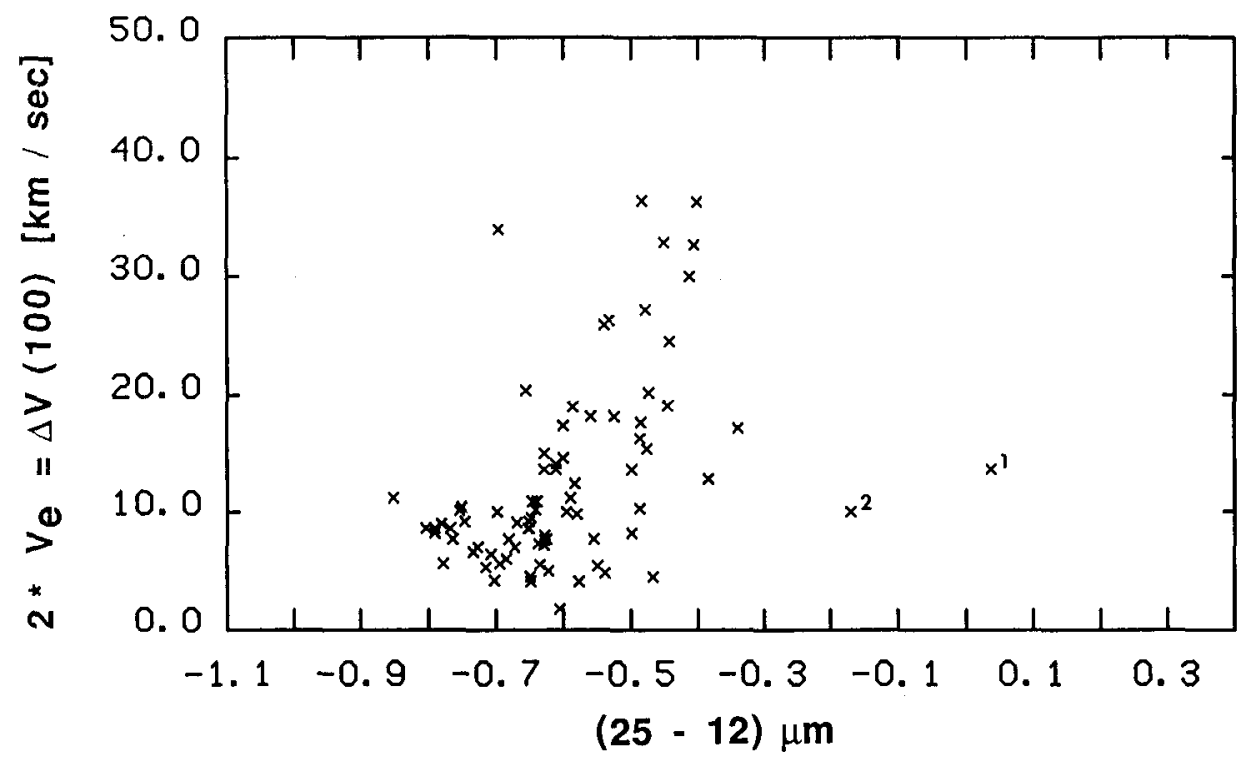

Figure 2: Shell expansion velocity (Ve) against (25-12) $\mu \mathrm{m}$ for sources with $|\mathrm{b}|>10^{\circ}$ within the Arecibo sky coverage. All widths are estimated from $1612 \mathrm{MHz}$ or mainline $\mathrm{OH}$ profiles at the profile peaks. Low mass stars have small expansion velocities until their shells become optically thick circa $(25-12) \mu \mathrm{m} \approx-0.5$, when they increase markedly. The outlying red sources, marked 1 and 2 , are respectively the PPN $18095+2704$ and 20531+2909 (Lewis et al 1990). 
from thick, red shells. This dichotomy is reinforced by the progressive concentration of color selected IRAS sources to the galactic plane with $(25-12) \mu \mathrm{m}$, and by their small Z-distributions. But these results are better explained by the notion that only the most massive stars reach the reddest colors, because they are the only ones capable of sustaining the requisite mass-loss. Indeed few stars with $|\mathrm{b}|>10^{\circ}$ have (25-12) $\mu \mathrm{m}>-0.4$. A contributory factor in this result is seen in Fig. 2, which shows a dramatic increase in the expansion velocities of shells from low mass stars, as these become opaque circa -0.5 . This contracts their red color range, so it needs a 3 times larger mass-loss rate for $(25-12) \mu \mathrm{m} \geq-0.3$ than otherwise obtains.

Figure 2 is perhaps the strongest direct evidence for evolution itself, since most stars with $|\mathbf{b}|>$ $10^{\circ}$ have a small (0.8-1.2 Mo) range of progenitor masses, and similar IR and masing detectability. They span the Mira sequence from the inception of mass-loss into a recognizable shell at (25-12) $\mu \mathrm{m} \approx-0.86$ to -0.4 . There is no mass segregation over this range. While the reddest end of the sequence is only populated by massive stars, the blue end has some too. These are sometimes optically identified supergiants with far IR signatures (Stencel et al 1989; Lewis 1991), and sometimes Miras, that are recognized as massive by their large SiO widths (Lewis 1991). The supergiants follow precisely the same color range as lower mass stars as they must have translucent shells to be recognized. Their color distribution incidentally executes a loop in the color plane, like that adduced above for the C-stars. Clearly the Mira sequence with $(25-12) \mu \mathrm{m}<-0.4$ is composed of stars with a wide range of masses: it is not primarily a progenitor-mass sequence.

The evolution of a circumstellar shell depends on changes to its mass-loss rate. This initially increases rather slowly, so there are far more sources with thin translucent shells than stars with thick shells. As the mass-loss rate increases, the dust shell thickens. This enhances its ability to shield molecules against interstellar UV photodegradation to a greater distance from the star, and permits a progressive addition of masing species with color. At the reddest colors the detectability of specific masing species is set by their individual excitation requirements. But on the final termination of mass-loss, the shell continues its expansion as a "fossil" shell, that only briefly remains a masing source, until its shielding is diluted by expansion. These stages are documented by the positions of sources in the IR two-color diagram.

This work was supported by NAIC, which is operated by Comell University under a management agreement with the National Science Foundation.

\section{References}

Baud, B., and Habing, H. J., 1983, A\&A.,127, 73.

Bedijn, P. J., 1988, A\&A., 205, 105.

Bowers, P.F., and Hagen, W. , 1984, Ap.J., 285, 637.

Epchtein, N., Le Bertre, T., and Lepine, J. R. D., 1990, $A \& A ., 227,82$.

Hacking, P. et al., 1985, Publ. Astr. Soc. Pac., 97, 616.

Harris, S., Clegg., P., and Hughes, J., 1988, Mon.Not.Roy. Astr.Soc., 235, 441.

Hrivnak, B. J., Kwok, S., and Volk, K. M., 1988, Ap J., 331, 832.

Kwok, S.,Hrivnak, B. J., and Volk, K. M., 1990, From Miras to Planetary Nebulae: Which Path for Stellar Evolution? ed M.O.Mennessier and A.Omont (Frontieres: Gif sur Yvette) p435.

Lewis, B. M. , 1991, AJ. 101, 254.

Lewis, B. M. , 1990, A.J. 99, 710.

Lewis, B.M., Eder, J. and Terzian, Y.,1990, ApJ. 362, 634.

Stencel, R. E., Pesce, J. E., and Bauer, W. H., 1989, A.J. 97, 1120.

van der Veen, W. E. C. J., 1989, A.\&A,.210, 127.

van der Veen, W., and Habing, H. J., 1988, A\&A., 194, 125.

Walker, H., et al., 1989, A.J., 98, 2163.

Willems, F. J. and de Jong, T., 1988, A.\&A.,196, 173. 